\title{
Los atolladeros de la economía latinoamericana
}

\author{
The difificulties of the Latin American economy*
}

Claudio Katz**

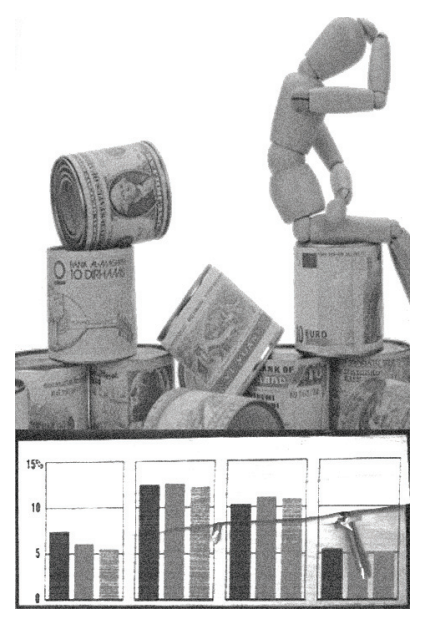

Resumen: El agravamiento de la crisis global acentúa las limitaciones de la economía latinoamericana para atemperar la turbulencia. Las enormes reservas terminarán socorriendo a los banqueros europeos si no hay avances en la moneda común. El extractivismo exportador impide repetir el desarrollo manufacturero asiático y refuerza la preeminencia de las empresas transnacionales. La batalla de los estudiantes chilenos ilustra cómo las resistencias sociales tienden a converger con la nueva oleada mundial de protesta juvenil.

Palabras-claves: América Latina. Crisis global. Neoliberalismo. Neodesarrollismo.

\begin{abstract}
The aggravation of the global crisis stresses the limitations of the Latin American economy to restrain the turbulence. The huge reserves ended up rescuing European bankers because there was no improvement in the common currency. The exporting extraction prevents the repetition of the Asian manufacturing development, and it strengthens the transnational companies' pre-eminence. The Chilean students' fight shows how the social resistances tend to converge on the new worldwide wave of young people's protest.
\end{abstract}

Keywords: Latin America. Global crisis. Neoliberalism. Neo-developmentalism.

* Este artículo "Los atolladeros de la economía latinoamericana", fue publicado en: El neoliberalismo y su crisis. Causas, escenarios y posibles desenvolvimientos, p. 112-126, ISBN.: 978-956-351-059-1, Santiago de Chile, 2012, ARCIS, REDEM.

** Economista, pesquisador, profesor. Miembro del EDI (Economistas de Izquierda). Su página web es: www.lahaine.org/katz. Correo: claudiokatz1@gmail.com. 
1 concluir el año 2011, reaparecen los nubarrones sobre la econo-
mía latinoamericana. El brusco agravamiento de la crisis global
augura un freno del crecimiento que aumenta el nerviosismo.
Durante el último quinquenio, el producto bruto regional mantuvo un ritmo ascendente del 5\% anual, a pesar de la desaceleración registrada en el 2009. La recuperación posterior se prolongó durante el 2011, y finalizaría con un incremento de 4,4\% del PBI. Hay previsiones de otro aumento del 4,1\% para el 2012, pero nadie sabe cuánto durarán los escudos protectores frente a la nueva turbulencia internacional.

Los neoliberales advierten contra la recaída y proponen recortar el gasto público para reforzar las defensas. Aunque la deuda pública y privada es muy inferior al promedio de los países desarrollados, promueven la contracción para asegurar las acreencias de los banqueros. Sus convocatorias a la austeridad expresan esta prioridad de los financistas.

Por el contrario, los heterodoxos alientan la continuidad de políticas contra-cíclicas. Los economistas de CEPAL presentan esta intervención como un acto de transgresión del neoliberalismo, olvidando que en numerosos países (México, Colombia o Chile), estas medidas complementan la continuidad del libre-comercio y las privatizaciones. Son iniciativas más dependientes de los ingresos fiscales que de las ideologías gubernamentales (Cepal-OIT, 2011).

Este intervencionismo no ha sido el único atenuante de la crisis. También la apreciación de las materias primas exportadas, el ingreso de capitales sin oportunidades de inversión en los países centrales y la desincronización del ciclo regional han limitado el impacto del temblor. (Katz, 2011)

Esta combinación de circunstancias se corrobora en la gran heterogeneidad de situaciones nacionales y en la escasa conexión de la reactivación con estrategias peculiares. Se han registrado altas tasas de crecimiento en países con políticas económicas heterodoxas (Argentina) y ortodoxas (Perú) y también resultados inversos en países del primer grupo (Venezuela) y del segundo (México). El efecto atenuado del tsunami global se ha verificado, además, especialmente en el sur del continente. Centroamérica y el Caribe sufren el duro contagio de la recesión estadounidense. 


\section{Dilemas estratégicos}

Frente a un probable escenario de recesión internacional se multiplican los cónclaves regionales. La frecuencia de estos encuentros contrasta, por ejemplo, con la pérdida de gravitación de las Cumbres Iberoamericanas. UNASUR ha logrado una centralidad inédita y comienza a operar como un MERCOSUR ampliado, incorporando a los países que suscribieron Tratados de Libre Comercio con Estados Unidos. El regionalismo sudamericano (Brasil y Argentina) tiende a converger con el área pro-norteamericana del Pacífico (Chile, Colombia, Perú). Esta coexistencia refuerza el predominio de proclamas, en desmedro de iniciativas concretas de integración.

Se discute, en primer lugar, la formación de un fondo de estabilización (FLAT) - a partir de ciertos mecanismos ya existentes (como el FLAR) — para auxiliar a las economías afectadas por corridas cambiarias. La fuga de divisas podría agravarse si los bancos y empresas extranjeras envían más dólares a sus casas centrales, para contrarrestar las situaciones de insolvencia. El FLAT está concebido como un instrumento de protección frente distintos escenarios de vaciamiento financiero. (Páez, 2011, p. 12; Kulfas, 2011, p. 12; Robba, 2011, p. 12)

Pero el monto de recursos comprometido en este resguardo (20.000 millones de dólares), sólo alcanzaría para socorros de emergencia en las economías pequeñas. Este tipo de reacciones defensivas ya se ensayaron en el pasado y no implicaron actos de solidaridad con las víctimas de la especulación. Al contrario, consolidaron una extranjerización del sistema bancario latinoamericano, que sería reafirmada si prospera la propuesta de asociar el FLAT con nuevos préstamos del BID.

En otros encuentros se debaten ideas para avanzar hacia la formación de alguna moneda común. La experiencia del Sucre - que utilizan Venezuela, Ecuador y Bolivia como unidad de cuenta para el intercambio comercial - es la referencia de estos proyectos. Ese signo permite reducir los costos de las transacciones, pero convive con el Dólar sin funcionar como moneda real. Aunque pretende incentivar un desacoplamiento de las divisas fuertes, no reemplaza el control de cambios, ni preserva a los países de los tormentosos flujos de capital. (Tovar, 2011, p. 12) 
El Sucre es una iniciativa más avanzada que los mecanismos de intercambio con billetes locales (Brasil-Argentina) o los convenios de pagos recíprocos (ALADI). Pero se encuentra muy lejos de sentar las bases de una moneda regional, basada en modelos de complementación solidaria opuestos a la centralización neoliberal que moldeó la gestación del Euro.

El Banco del Sur es otro termómetro de la parsimonia que domina en los proyectos de integración. Ya han transcurrido varios años desde su constitución formal y aún faltan tres confirmaciones parlamentarias de los siete suscriptores del proyecto. Nadie define el destino de los créditos y el capital comprometido para la entidad es muy reducido, en comparación a un gigante de la zona como es el BNDES de Brasil (Brasil, Venezuela y Argentina aportarían cada uno, 2.000 millones de dólares al nuevo Banco, Ecuador y Uruguay sumarían 400 millones y Bolivia y Paraguay contribuirían con 100 millones).

Pero el tema más relevante ocupa poco espacio en la reflexión regional. ¿Qué hacer con las enormes reservas que acumula América Latina? Como resultado del superávit comercial y la afluencia de divisas, los Bancos Centrales ya atesoran 574.000 millones de dólares. Se ha creado un excedente que contrasta con la enfermedad de vaciamientos sufridos por la zona en los momentos de crisis. ¿Los nuevos recursos respaldarán inversiones productivas coordinadas? ¿O se dilapidarán en acciones que perpetúan la dependencia?

La actual indefinición conduciría a la desaparición de los fondos por la misma ruta que ingresaron. La Unión Europea, el gobierno norteamericano y el FMI intentan canalizar las reservas hacia un socorro del sistema financiero mundial. Presentan este auxilio como un "aporte de América Latina" a las economías avanzadas, olvidando la deuda histórica que arrastra el Primer Mundo con la región. Proponen acompañar la compra de títulos europeos que realizarían China y otros BRICS, para apuntalar los bancos quebrados. La adquisición de estos papeles acrecentaría las cuantiosas inversiones que ya tiene Latinoamérica, en esa modalidad de colocaciones.

La participación de Brasil en la cartera del FMI constituyó un primer guiño hacia este nuevo compromiso. En la última reunión del G 20 (Cannes) se reforzó este curso mediante explícitas exigencias de intermediación del cuestionado organismo, en cualquier auxilio financiero a Europa. Este idilio del gobierno 
brasileño con el FMI no es un dato menor, si se tiene en cuenta que el país es el quinto poseedor internacional de Bonos del Tesoro estadounidenses (en diciembre del 2010, Brasil sólo era superado en esas acreencias por China, Japón, Gran Bretaña y los países árabes exportadores de petróleo. Ver Bandeira, 2011).

El dinero que se use para rescatar a los financistas europeos será sustraído del FLAT, la moneda común, el Banco del Sur y la integración productiva. Sería el nuevo precio que pagarían Argentina, México y Brasil para continuar participando en el G 20, con iniciativas que reafirman la asociación de las clases dominantes locales con el establishment global. Esta orientación se ubica en las antípodas de dos medidas insoslayables para avanzar hacia una integración regional progresista: la nacionalización de los bancos y la estricta regulación de los flujos de capital.

Estas acciones son indispensables en la coyuntura actual, para definir respuestas conjuntas ante contradictorios procesos de revalorización y desvalorización monetaria. La región ha padecido en los últimos años los efectos adversos del ingreso de dólares (que sobrevaluan la moneda local) y también del egreso de divisas (que provocan las conocidas tensiones cambiarias).

Seguramente Brasil definiría el rumbo a seguir, desde el momento que maneja entre el 50 y el $60 \%$ de las reservas totales. Ya actúa como subpotencia, adaptando el Mercosur a un juego multilateral, basado en coordinaciones estratégicas con Estados Unidos. Esta política deja poco espacio para la formación de un fondo financiero latinoamericano (ver Berterretche, 2011).

El terremoto sufrido por el Euro reforzaría, además, la aversión de los gobiernos brasileños a repetir en la región, el papel jugado por Alemania en el Viejo Continente. Si la gran potencia germana quedó hundida en el atolladero de la Unidad Europea, Brasil tiene menos posibilidades de liderar una integración capitalista de Sudamérica.

\section{Las consecuencias del extractivismo}

La gran dependencia regional del vaivén internacional de los precios de las materias primas acrecienta las críticas hacia el modelo exportador. Este esquema 
incentiva la multiplicación de emprendimientos exclusivamente destinados a comercializar productos básicos. Mientras crece la influencia del agro-negocio, las inversiones extranjeras consolidan la especialización petrominera. Todas las potencias buscan asegurarse el aprovisionamiento de insumos latinoamericanos, afianzando la inserción de la zona como granja o socavón de la economía mundial.

El término "extractivismo exportador" — que muchos analistas utilizan para describir este modelo - ofrece un acertado retrato del esquema actual. Destaca las nefastas consecuencias de la minera contaminante y la agricultura de exportación, en desmedro del abastecimiento interno ("Articulación de movimientos sociales hacia el ALBA”, Buenos Aires, 16/ago./2011).

Este curso extractivo potencia la vulnerabilidad de América Latina. sin generar necesariamente procesos de "reprimarización" o "desindustrialización". Pero impone senderos frontalmente opuestos al desarrollo manufacturero que ha seguido el Sudeste Asiático. La atadura a las exportaciones básicas suscita, además, permanentes interrogantes sobre la continuidad del ciclo alcista de las materias primas que comenzó en el 2003 y perdura hasta la actualidad.

Algunas explicaciones atribuyen esta valorización a los movimientos especulativos y a la falta de supervisión de los mercados agrícolas de futuro. La desregulación de esta plaza facilitó el ingreso de los bancos de inversión al negocio y la consiguiente presencia de un mortífero arsenal de derivados. El uso de instrumentos financieros en el sector aumentó de 500.000 millones (2000) a 13 billones de dólares (2008). (Ver Munevar, 2011)

Otras caracterizaciones destacan cómo el incremento del precio del petróleo potenció la expansión de los agro-combustibles y señalan que el 12\% de la producción mundial de maíz ya se destina a la fabricación de etanol. Una tercera explicación estima que la demanda china ha establecido un nuevo piso de cotizaciones para todas las materias primas.

Esta diversidad de interpretaciones alude, en los hechos, a distintos procesos temporales. Mientras que las maniobras financieras determinan los incrementos coyunturales de precios, los agrocombustibles y las compras asiáticas inciden sobre el mediano y el largo plazo.

El boom de las commodities ha reabierto también viejas controversias teóricas sobre el deterioro de los términos de intercambio y la influencia de las 
exportaciones primarias en el subdesarrollo latinoamericano. Pero cualquiera sean las respuestas a estos interrogantes son evidentes los efectos sociales nocivos del extractivismo. Sólo el desarrollo manufacturero permitiría crear los puestos de trabajo necesarios para erradicar el atraso de la región. Este salto se encuentra impedido por la dominación que ejercen las empresas transnacionales de la economía latinoamericana.

Ese predominio determina incluso muchas posturas de política exterior. Cuándo Brasil y Argentina rechazan el G 20 la regulación de los precios de los alimentos, siguen el mandato de las grandes compañías. La hambruna de los empobrecidos no es analizada con criterios de solidaridad, sino como una oportunidad de negocios. El extractivismo perpetúa el sometimiento del ciclo latinoamericano a la tiranía de la reproducción dependiente. Esta subordinación tiene mayor impacto actual en la esfera comercial o productiva, que en el área tradicional del endeudamiento. Pero la experiencia indica que la atadura a las exportaciones básicas termina recreando el agobio financiero.

\section{Desigualdad y explotación}

Algunos economistas ponderan el rumbo actual destacando la caída de la desocupación que acompaña al crecimiento. Pero las cifras sólo indican reducidos cambios acordes al vaivén del ciclo. En la desaceleración del 2009 la tasa de desempleo llegó al 8,1\%, luego bajó al 7,3\% (2010), este año rondaría el 7\% y si se confirman los augurios de freno volvería a subir.

Pero lo más relevante es la baja calidad de los nuevos empleos, reclutados en su gran mayoría en el sector informal. La precarización se mantiene como una norma, tanto de la recesión como de la prosperidad. Este deterioro complementa la degradación que imponen la disminución de las remesas, el éxodo rural y la marginalidad urbana. Millones de individuos están condenados a formas de supervivencia infra-humana, que logran visibilidad mediática sólo en los momentos de gran cataclismo (incendio de una cárcel superpoblada, alud en una favela, inundaciones en zonas desprotegidas).

La manifestación más dramática de este infierno en la región centroamericana es la expansión del narcotráfico. Esta actividad es un refugio de super- 
vivencia para los campesinos endeudados y para los jóvenes desempleados incorporados a la delincuencia organizada. El macabro curso de la guerra emprendida por el gobierno de México ya cobró la vida de 50.000 personas. Con un estado de excepción permanente se legaliza la brutalidad criminal que ejercitan las mafias y sus adversarios-cómplices del estado (Petras, 2010).

La magnitud de las fortunas en juego es proporcional a la sangría de un negocio que manejan los clanes de la lumpen-burguesía. Este término se utilizó erróneamente en el pasado para retratar en forma indiscriminada a las clases dominantes latinoamericanas. Pero en realidad tipifica sólo a un sector muy específico, que ha transnacionalizado, diversificando y blanqueando las ganancias obtenidas en los circuitos paralelos de la acumulación. La burguesía lumpen está muy entrelazada con sus pares del sector formal, pero no integra el club estable de los grandes dominadores de la región.

Es sabido que el desgarramiento social padecido en América Latina recrea la pobreza y la desigualdad. Sin embargo algunos analistas celebran la escasa reducción del nivel de inequidad registrado durante el reciente ciclo de crecimiento. Olvidan que América Latina continúa encabezando todos los récords internacionales de polarización social. La región incluye a cuatro de los países que lideran este vergonzoso indicador (Colombia, Bolivia, Honduras, Brasil). El coeficientes de Gini que mide la desigualdad ilustra un promedio zonal $(51,6)$ muy superior a media mundial $(39,5)$.

En este terreno la principal novedad radica en la generalización de las políticas asistenciales, que tienden a atenuar los explosivos efectos de la fractura social. Todas las administraciones implementan estos planes como imperativos de gobernabilidad. El costo de estas acciones se asemeja en todos los países y es muy reducido en proporción al producto bruto.

En Argentina rige la asignación universal ( $0,40 \%$ del PIB), en Brasil la Bolsa Familia $(0,47 \%)$, en México el programa Oportunidades $(0,51 \%)$, en Bolivia el Bono Juancito Pinto (0,33\%), en Venezuela las Misiones $(0,45 \%)$, en Ecuador el Bono de Desarrollo Humano (1.17\%), en Chile el Plan Solidario $(0,11 \%)$, en Colombia la Iniciativa Familias $(0,39 \%)$ y en Perú el Proyecto Juntos $(0,14 \%)$ (Gonçalves, 2011).

Este tipo de asistencias protege a los desamparados, pero no genera ninguna redistribución del ingreso. En la medida que coexiste con la precarización 
laboral tiende más bien a convalidar la segmentación del mercado de trabajo. Esta fractura acentúa las viejas modalidades de superexplotación que caracterizan al capitalismo latinoamericano. Las empresas foráneas lucran en las maquilas con la baratura de la fuerza laboral y los capitalistas locales exprimen a los asalariados, para compensar su escasa relevancia en el mercado global.

\section{Neoliberalismo y neo-desarrollismo}

En América Latina comienza a verificarse cierto viraje en el pensamiento dominante, puesto que el neoliberalismo ha quedado desprestigiado por el resultado de sus gestiones. Prometió un gran despegue con privatizaciones y desregulaciones y terminó reforzando los viejos desequilibrios de la reproducción dependiente. Luego estimuló el endeudamiento para paliar estos trastornos y precipitó tormentosos estallidos financieros.

Aunque numerosos gobiernos preservan esta misma estrategia con algún ajuste cosmético, otras administraciones comienzan a sustituir el credo neoliberal por planteos neodesarrollistas. Esta propuesta gana adherentes con discursos de intervención del estado, cuestionamientos a la pérdida de competitividad cambiaria ("enfermedad holandesa") y convocatorias a imitar el camino asiático de industrialización (este programa ha sido expuesto en el encuentro "Crecimiento con estabilidad financiera y el nuevo desarrollismo". Ver también Gaitán y Boschi, 2010).

Pero este renacer desarrollista no es preponderante aún en el país más industrializado. Brasil mantiene su primacía manufacturera en la región, pero con pérdidas de posiciones frente al agronegocio. Las políticas oficiales de subsidios fabriles no compensan la apreciación de la moneda y el sistemático incremento de la tasa de interés. El país no cuenta con recursos, tecnología o mercados suficientes para compatibilizar el modelo alemán, que combina la severidad financiera con la competitividad productiva. Por esta razón se intensifican las tensiones entre la ortodoxia monetarista y los planteos industrialistas. El país se afianza como exportador de materias primas y su participación en el producto industrial mundial ha decaído de 2,9\% (1980) a 1,9\% (en la década 
pasada). La tasa de inversión industrial es baja (20\%) y el porcentaje de exportaciones manufactureras decayó frente a las materias primas que concentran el $80 \%$ de las ventas externas (Pochman, 2011). El principal promotor del viraje neodesarrollista es Luiz Carlos Bresser Pereira (Globalizacão e competição, Folha de S.Paulo, 2-22-09).

Argentina ha puesto en marcha un intento neodesarrollista más sostenido. Este ensayo constituye una reacción frente al descomunal desplome sufrido durante el cenit neoliberal. El gobierno ha buscado recomponer la gravitación de la burguesía industrial, en desmedro de los bancos y en conflicto con el agro-negocio. Pero este último sector ha capitalizado su actividad y se niega a compartir las enormes rentas que acapara. Además, la burguesía industrial perdió peso por la extranjerización y sostiene sus ganancias con subsidios y remarcaciones de precios. Estas presiones neutralizan el proyecto re-industrializador (los neodesarrollistas se agrupan en torno al denominado "Plan Fénix" y su teórico más renombrado es Aldo Ferrer - Ferrer, 2010. Hemos expuesto una caracterización reciente en Katz, 2011).

La industria mexicana presenta otro panorama al quedar amoldada (a través de las maquilas) a las líneas de fabricación estadounidenses. Los replanteos desarrollistas implican en este caso confrontar con una estrategia de libre comercio con la primera potencia, que ha desarticulado el viejo entramado industrial centrado en el mercado interno (Vidal y Guillén, 2007).

El resurgimiento neo-desarrollista se encuentra obstruido en América Latina por el predominio del extractivismo, la extranjerización de la economía y el desplazamiento de las viejas burguesías nacionales por nuevos grupos exportadores. Estas limitaciones son habitualmente omitidas por quiénes observan esa estrategia como la más conveniente, o la única factible en la actualidad.

El neodesarrollismo incluye también un ala más progresista, que reconoce la ausencia de clases capitalistas dispuestas a asumir la conducta clásica del industrialismo (inversión, riesgo, competencia). Proponen compensar esta orfandad del sujeto burgués con políticas sustitutivas de inversión y gerenciamiento público.

Esas medidas no son concebidas en una dirección pos-capitalista, sino como acciones tendientes a remodelar el orden social vigente. Por eso implican 
no sólo acciones de regulación estatal, sino también fuertes subsidios a los grupos empresarios que se desea promover como protagonistas de la vida económica. ¿Pero qué beneficios aportarían estas subvenciones a la mayoría de la población? ¿Por qué razón los trabajadores y ciudadanos deberían solventar el fortalecimiento de un régimen social que no les pertenece?

Es importante indagar estas contradicciones para clarificar el significado contemporáneo del nuevo desarrollismo. Algunos autores críticos no observan mayores diferencias con neoliberalismo. Consideran que el cambio de retórica encubre la continuidad de ofensivas del capital sobre el trabajo o la introducción de regulaciones para auxiliar a los bancos.

Pero conviene evaluar esos parentescos en función de los intereses en juego. Si el libre-comercio es la ideología de los agroexportadores y la ortodoxia monetaria opera como credo de los banqueros, la defensa de los subsidios forma parte del guión industrialista. El neodesarrollismo adapta esta última tradición a las necesidades actuales de los grupos fabriles más concentrados, transnacionalizados y exportadores (compañías "Multilatinas"). (Dos interpretaciones críticas del neodesarrollismo en: Azcurra, 2011 y en Sepla, 2011.)

Estas empresas tienden a expandirse hacia las economías vecinas para contrarrestar la estrechez de los mercados internos de origen. Compensan esta limitación con inversiones de alta rentabilidad en el exterior. La forma en que Petrobras bloqueó la nacionalización de los combustibles en Bolivia es un ejemplo de esta conducta. (Mediante presiones directas de Lula se desnaturalizaron los contratos, se congeló el aumento de la tributación y quedaron anuladas las auditorias. Petrobras volvió a cotizar en Bolsa anotando como propias las reservas del Altiplano y neutralizó los proyectos de transferir la industrialización del gas de São Paulo a Bolivia. Ver Solíz Rada, 2011.)

Las víctimas de esta política suelen utilizar el término "sub-imperialismo" para caracterizar este comportamiento. También aplican este concepto para retratar acciones del ejército brasileño en Haití, que repiten estrategias de militarización ya ensayadas en las favelas. La denominación no sólo tiene un legítimo propósito de denuncia. También induce a revitalizar un concepto, que debería ser estudiado mediante comparaciones con el uso inicial que tuvo en los años 60. 
Es muy importante precisar el sentido de cada noción para caracterizar en forma adecuada el alcance regional de la turbulencia económica actual. El término crisis es por ejemplo utilizado con tantas acepciones, que a veces resulta imposible discernir si alude a una coyuntura, a una etapa o a un devenir del capitalismo. Tampoco se sabe si evalúa una situación global, regional o nacional.

En esta utilización tan polisémica tampoco se suele aclarar, si se está analizando un ciclo económico. En este caso, el término crisis debería ser referido a situaciones de recesión, contrapuestas al crecimiento. Si cuando cae el PBI y el empleo hay crisis y cuando aumentan ambas variables también hay crisis, resulta imposible entender de qué se está hablando. La clarificación de los debates es una deuda pendiente en el pensamiento crítico latinoamericano, cuya resolución permitirá definir con mayor exactitud la etapa actual.

\section{El escenario político}

Las tendencias económicas comunes de América Latina se procesan en distintos contextos políticos de gobiernos derechistas, centroizquierdistas y reformistas. Estas administraciones actúan, a su vez, en variados marcos de conquistas o repliegues populares. Las semejanzas estructurales entre Colombia y Venezuela quedan replanteadas a la hora de observar quién gobierna y lo mismo vale para México y Argentina o para Guatemala y Bolivia. El devenir de la economía regional depende de los desenlaces políticos en cada país.

Durante el bienio 2010-11 los gobiernos derechistas enfrentaron múltiples problemas. El imperialismo norteamericano perdió a su agente directo en Perú y observa con gran inquietud la impotencia de su socio mexicano para lidiar con el narcotráfico. La violencia facilitó el regreso del militarismo conservador en Guatemala y las matanzas de los paramilitares continúan en Colombia. Pero en todos los casos crece el hastío de la población. Los golpistas hondureños debieron replegarse, buscado un compromiso con el presidente depuesto y el reaccionario gobierno de Chile afronta desventuras económicas, fracasos de gestión y gran resistencia social. 
Es evidente, además, que las pesadillas afrontadas por el Pentágono en el Medio Oriente reducen la capacidad de intervención de la IV flota y de los marines desplegados en Colombia. Por esta razón, las campañas de intimidación se procesan a través de los grandes medios de comunicación, que definen en cada momento a quién hostilizar y a quién bendecir. La derecha se mantiene muy activa, pero sin recuperar la iniciativa que tuvo durante el cenit del neoliberalismo.

Los principales ganadores de la coyuntura son presidentes de centroizquierda como Dilma Rouseff y Cristina Fernández Krichner, que obtuvieron arrolladores triunfos en los comicios. En ambos casos el oficialismo revalidó títulos, incorporando sectores medios y altos a su base electoral. Mientras que en Brasil la victoria se consumó en un clima de pasividad conservadora y despolitización, en Argentina han prevalecido las tensiones con la derecha, la participación de los movimientos sociales y la renovada politización de la juventud.

Uruguay sigue el modelo brasileño de buena letra hacia los capitalistas y desatención de las demandas sociales y también el nuevo mandatorio de Perú busca recrear el sendero social-liberal inaugurado por Lula. El limitado impacto que hasta ahora tuvo la crisis global en Sudamérica ha contribuido a este afianzamiento del centro-izquierdismo.

Pero lo más llamativo es la creciente atracción que ejerce esa referencia sobre los gobiernos más radicales de Venezuela, Bolivia (y en cierta medida Ecuador). Estas administraciones surgieron confrontando con el imperialismo, impulsando movilizaciones populares y promoviendo reformas democráticas y sociales. Ahora enfrentan encrucijadas que determinarán su futuro.

\section{Definiciones en el eje radical}

La economía venezolana ha sido más afectada por la crisis que el promedio sudamericano. Los desbalances tradicionales (dependencia de la factura petrolera, bajísima producción local, alto nivel importaciones, consumo suntuario) condujeron a nuevas devaluaciones para atemperar el déficit fiscal, en un 
marco de alta inflación. Las medidas progresistas (nacionalización del oro) continúan coexistiendo con el favoritismo hacia la "boliburguesía" y el respiro logrado con ciertas acciones reformistas, no resuelve los problemas de una economía periférica muy saboteada por las clases dominantes.

Aunque la derecha se entusiasmó con la enfermedad de Chávez, la popularidad del presidente persiste y no será fácil impedirle otra renovación de su mandato. El estancamiento del proceso bolivariano obedece más a sus propias contradicciones, que al acoso de la reacción (Aharonian, 2011).

Tanto la entrega de varios militantes de la insurgencia al gobierno colombiano, como el apoyo a dictadores árabes (especialmente de Siria) suscitan malestar. Si la profundización del proceso bolivariano continúa posponiéndose, este proyecto quedará congelado y comenzará a equiparse con los restantes gobiernos de centroizquierda.

La misma disyuntiva afronta Bolivia. La estatización de los hidrocarburos quedó reducida y persisten los privilegios de las compañías extranjeras. La reforma agraria sigue pospuesta y las mejoras populares no están a tono con la derrota del neoliberalismo. La masiva resistencia al incremento de precios del combustible ("gasolinerazo") fue la primera advertencia de este estancamiento. Un segundo choque con pueblos de la selva — que se oponían a la construcción de una carretera- tuvo un corolario represivo brutal.

Las medidas descolonizadoras que acompañaron al establecimiento del estado plurinacional son incompatibles, con el perfil autoritario que está adoptando el oficialismo. Bolivia no puede prescindir de sus reservas minerales para erradicar el atraso, pero la utilización de estos recursos requiere respetar el medio ambiente, evitar el monocultivo, desarrollar el cooperativismo y compatibilizar en consultas democráticas la multitud de intereses populares en conflicto. El logro de estos objetivos exige, a su vez, abandonar la estrategia de gestar un "capitalismo andino amazónico" (ver Almeyra, 2011; Zibechi, 2011; Manifiesto de la Coordinadora Plurinacional de la Reconducción, 2011.)

Las mismas contradicciones presentan mayor dimensión en Ecuador. El gobierno ha demostrado firmeza frente a las agresiones norteamericanas, pero 
continúa confrontando con el movimiento indígena, desconoce las propuestas de preservación de los recursos naturales y pospone la implementación de transformaciones socio-económicas significativas.

El techo de logros que están encontrando los gobiernos radicales repercute directamente sobre el ALBA. Este organismo ha quedado desdibujado frente a UNASUR y sus iniciativas han perdido el impacto inicial que tuvo la creación de TELESUR, la formación de PETROCARIBE, la solidaridad con Cuba, los emprendimientos de salud y alfabetización o el apoyo antiimperialista a Honduras y Haití. El mismo apaciguamiento afecta al proyecto del socialismo del siglo XXI, que tiende a diluirse en ausencia de estrategias de radicalización anticapitalista.

El futuro del ALBA quedará también signado por el resultado de las reformas económicas que han comenzado en Cuba. En una isla con muy pocos recursos no hay pobreza o criminalidad, sino una gran cobertura de las necesidades básicas en un marco de significativa escasez. En lugar de analfabetismo, deserción escolar y mortalidad infantil hay dificultades para continuar el sostenimiento de la educación y la salud gratuitas.

Cuba padece la asfixia comercial del bloqueo y las graves adversidades coyunturales que han generado la caída del precio del níquel, los menores ingresos del turismo y los huracanes. Una economía con alta calificación de la mano de obra carece de industria o agricultura productivas y luego del colapso de la URSS debió sobrevivir mediante el turismo, las remesas, el doble mercado y los convenios con empresas extranjeras. Junto a la errónea subsistencia del modelo de estatización integral apareció un importante flujo de divisas, que no se transforma en inversión. Los proyectos para incentivar la actividad mercantil apuntan a contrarrestar esta asfixia, recomponiendo la productividad y reduciendo la dependencia de los alimentos importados.

El gran desafío será implementar esta política sin permitir el retorno al capitalismo. Cuba ya salió airosa en el pasado de gestas que parecían irrealizables (período especial, bloqueo, invasiones) y puede alcanzar las nuevas metas con participación popular, democratización y limitaciones a la desigualdad social. El futuro del ALBA y los proyectos de renovación del socialismo dependen en gran medida de ese proceso. 


\section{El empalme con los indignados}

¿Cuál será el efecto de la crisis global sobre las luchas sociales de América Latina? Las resistencias alcanzaron un pico de intensidad durante las rebeliones del 2000-05, que tumbaron a varios gobiernos reaccionarios. Estos levantamientos indujeron a las clases dominantes a actuar con mayor cautela frente al ajuste, tanto en los epicentros como en los vecindarios de las sublevaciones. Las movilizaciones posteriores han sido más acotadas (defensa del salario, el empleo o los recursos naturales), con la excepción de la resistencia casi insurreccional que se registró contra el golpe en Honduras (un balance general de las resistencias en Seoane; Taddei; Algranati, 2011).

Las batallas de los últimos años han estado a tono con el alcance acotado que tuvo el descalabro mundial sobre la región. Esta reacción se acrecentaría si aumenta ese impacto, pero esta vez podría incorporar un novedoso empalme con la oleada de protesta que comienza a notarse en todos los continentes.

Las reacciones puramente defensivas que rodearon en el 2008 al debut de la crisis han quedado modificadas por la primavera que conmovió al mundo árabe. Las batallas que se libran en Grecia, la irrupción de los indignados españoles, el descontento social en Inglaterra, las huelgas en Italia y los plebiscitos de Islandia ilustran este cambio de clima. La nueva generación ha convertido las redes sociales en un instrumento de organización que cruza las fronteras e incentiva a los ocupantes de Wall Street. La jornada mundial del 15 de octubre pasado reunió a millones de manifestantes en 950 ciudades de 80 países.

Esta nueva tónica no tardará en contagiar a la región latinoamericana. La extraordinaria movilización de los estudiantes chilenos podría constituir la primera expresión de esta nueva oleada. Los universitarios y secundarios trasandinos no sólo confrontaron con un gobierno derechista que oscila entre la represión y el vaciamiento de las negociaciones. También conquistaron la simpatía popular, con formas de acción que retoman el viejo formato de la alianza obrero-estudiantiles de los 70. "Nuestros hijos no pasarán de curso pero pasarán a la historia", destacaban las pancartas de las marchas que plantearon una demanda explosiva para el neoliberalismo. La exigencia de gratuidad para la 
educación desenmascara el escandaloso endeudamiento que padecen los estudiantes y ataca un cimiento de la desigualdad social.

Las resistencias en curso cuestionan no sólo a los banqueros y al neoliberalismo, sino al propio sistema capitalista. Se ha puesto a la orden del día definir quién pagará los terribles costos de la crisis y frente a esta disyuntiva, vuelve a enhebrarse el tejido de solidaridades de América Latina con los pueblos del Primer Mundo.

Recebido em 3/8/2012 - Aprovado em 13/8/2012

\section{Referências bibliográficas}

“Articulación de movimientos sociales hacia el ALBA", Buenos Aires, 16/ago./2011. AHARONIAN, Aram. "Venezuela: dos meses para atravesar un campo minado", ALAI,06/oct./2011.

ALMEYRA, Guillermo. "Bolivia: el desarrollismo contra el desarrollo", La Jornada, 02/oct./2011.

AZCURRA, Fernando Hugo. Las diez tesis sobre el nuevos desarrollismo elaboradas por economistas heterodoxos". Disponible en: <www.pctargentina.org/>, feb. 2011.

BANDEIRA, Luiz Alberto Moniz. "A crise afecta a todos os países inclusive Brasil”, ALAI, 29/ago./2011.

BERTERRETCHE, Juan. “¿Acuerdo económico defensivo?”, 19/ago./2011. Disponible en $<$ http://www.kaosenlared.com $>$.

BRESSER PEREIRA, Luiz Carlos. "Globalização e competição", Folha de S.Paulo, 02/fev./2009.

CEPAL-OIT. Coyuntura laboral en América Latina y el Caribe, jun. 2011, n. 5. Disponible en: $<$ http://www.oit.org.pe/>

ENCUENTRO "Crecimiento con estabilidad financiera y el nuevo desarrollismo". Disponible en: <http://www.tenthesesonnewdevelopmentalism.org>.

FERRER, Aldo. “El nuevo desarrollismo”, en Miradas al Sur, 06/nov./2010.

GAITÁN, Flavio; BOSCHI, Renato. "América Latina recupera el pensamiento desarrollista", El Clarín, 21/dic./2010. 
GONÇALVES, Reinaldo. Redução da desigualdade da renda no governo Lula. Análise comparativa, 20/jun./2011. Disponible en: <http://www.ie.ufrj.br $>$.

KATZ, Claudio. "A 10 años del 2001: los economistas debate", Revista Kamchatka, n. 7, año 4. Buenos Aires: FCE-UBA, oct. 2011a.

KATZ, Claudio. "Los atolladeros de la economía latinoamericana", en: El neoliberalismo y su crisis. Causas, escenarios y posibles desenvolvimientos. Santiago de Chile: 2012, p. 112-126. (ISBN 978-956-351-059-1) ARCIS, REDEM.

KATZ, Claudio. "The singularities of Latin America". The crisis and the left, Socialist Register. Toronto, v. 48, 2012.

KULFAS, Matías. “Que integración regional conviene”, 20/jun./2011, p. 12.

MANIFIESTO de la Coordinadora Plurinacional de la Reconducción, 13-7-2011.

MUNEVAR, Daniel. Alzas en los precios de alimentos. Una mirada desde América Latina, CADTM, jun. 2011. Disponible en: <http://www.cadtm.org/>.

PÁEZ, Pedro. "Detrás del desorden económico mundial," 3/oct./2011, p. 12.

PETRAS, James. "Latin America: Road to 21st. century capitalist development", 25/ oct./2010. Disponible en <http://www.ccun.org $>$.

PLAN FÉNIX. Disponible en <www.econ.uba.ar>.

POCHMANN, Marcio. "O Brasil e a nova desindustrialização”, 09/jun./2011. Disponible en: <www.centrodeestudossindicais.wordpress.com/>.

ROBBA, Alejandro. "El desafío de la integración financiera regional”, 07/ago./2011, p. 12.

SEOANE José; TADDEI, Emilio; ALGRANATI, Clara, "Tras una década de luchas". Buenos Aires: Herramienta, 2011. n. 46.

SEPLA. "Declaración de Guararema", Sociedad Latinoamericana de Economía Politica y Pensamiento Crítico. Guararema, jun. 2011.

SOLÍZ RADA, Andrés. "Entrevista" Pueblos. Revista de información y debate. Disponible en: <www.revistapueblos.org/>, 27/oct./ 2011.

TOVAR, Eudomar. Consejo Monetario del ALBA, "No podemos evitar el impacto", 03/oct./2011, p. 12.

VIDAL, Gregorio; GUILLÉN, Arturo. "La necesidad de construir el desarrollo en América Latina", Repensar la teoría del desarrollo en un contexto de globalización. Buenos Aires: CLACSO, 2007.

ZIBECHI, Raúl. “La obstinada potencia de la descolonización”, ALAI, 29/sept./ 2011. 